\title{
Enzalutamide promotes mesenchymal plasticity via Snail activation
}

Snail was
the only
consistently
upregulated
EMP driver
at both the
mRNA and
protein levels

Epithelial-to-mesenchymal plasticity (EMP), a process linked with the development of both metastases and drug resistance, is known to be involved in the progression of prostate cancer, although the mechanisms that mediate this effect are poorly understood. Now, new data from in vitro and in vivo models indicate that enzalutamide, a widely used form of androgen deprivation therapy, de-represses the EMP-promoting transcription factor SNAI1 (also known as Snail).

Researchers found that enzalutamide induced the expression of several markers of EMP, including Snail, in cells of the C4-2 prostate cancer cell line. This effect was then confirmed to be AR-mediated by the observation that androgens reverse the effects of enzalutamide on Snail expression, and that AR knockdown attenuates both of these effects. The importance of Snail to EMP was also confirmed in other prostate cancer cell lines (such as LAPC4 and 22Rv1), in which Snail was the only consistently upregulated EMP driver at both the mRNA and

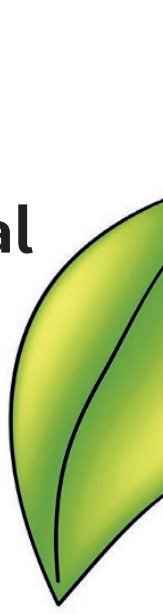
protein levels.

The role of Snail as an inducer of enzalutamide-mediated EMP was then demonstrated by knockdown of Snail expression in C4-2 cells, which abrogated the ability of enzalutamide to induce cellular motility.

Importantly, researchers were able to confirm that transcription of Snail is under the direct control of the AR using luciferase reporter assays, which indicated that the AR is recruited to three separate regions of the Snail promoter.

The role of Snail in metastatic castration-resistant prostate cancer (mCRPC) was then monitored over an extended period in a single patient receiving enzalutamide.
2

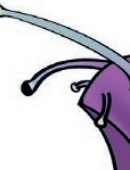

दे r
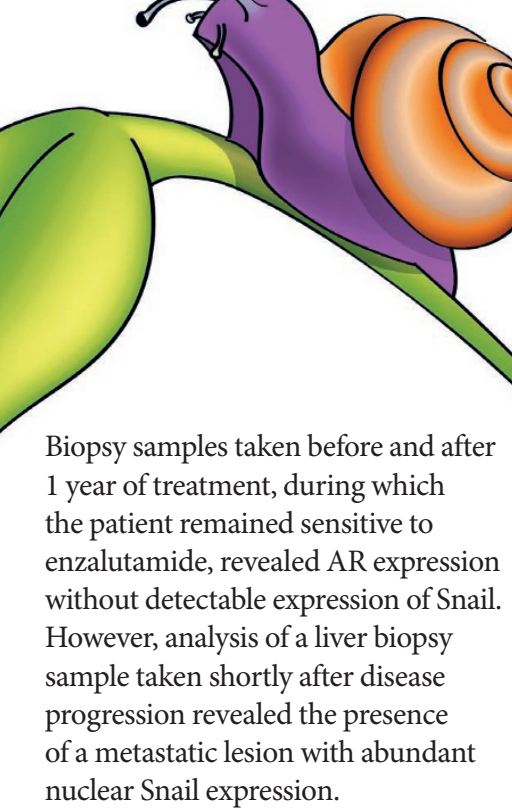

Biopsy samples taken before and after 1 year of treatment, during which the patient remained sensitive to enzalutamide, revealed AR expression without detectable expression of Snail. However, analysis of a liver biopsy sample taken shortly after disease progression revealed the presence of a metastatic lesion with abundant nuclear Snail expression.

These findings highlight the rationale for targeting proteins involved in the EMP process in order to avoid or further delay the onset of mCRPC in patients with prostate cancer.

Peter Sidaway

ORIGINAL ARTICLE Miao, L. et al. Disrupting androgen receptor signaling induces Snailmediated epithelial-mesenchymal plasticity in prostate cancer. Cancer Res. http://dx.doi.org/ 10.1158/0008-5472.CAN-16-2169 (2017) 